Design and operating characteristic of a vacuum furnace for time-of-flight inelastic neutron scattering measurements

J. L. Niedziela, R. Mills, M. J. Loguillo, H. D. Skorpenske, D. Armitage, H. L. Smith, J. Y. Y. Lin, M. S. Lucas, M. B. Stone, and D. L. Abernathy

Citation: Review of Scientific Instruments 88, 105116 (2017);

View online: https://doi.org/10.1063/1.5007089

View Table of Contents: http://aip.scitation.org/toc/rsi/88/10

Published by the American Institute of Physics

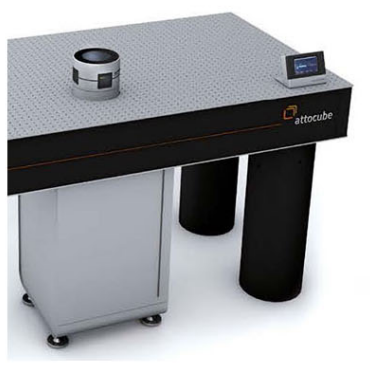




\title{
Design and operating characteristic of a vacuum furnace for time-of-flight inelastic neutron scattering measurements
}

\author{
J. L. Niedziela, ${ }^{1,2, a)}$ R. Mills, ${ }^{3}$ M. J. Loguillo, ${ }^{3}$ H. D. Skorpenske, ${ }^{3}$ D. Armitage,${ }^{3}$ H. L. Smith, ${ }^{4}$ \\ J. Y. Y. Lin, ${ }^{3}$ M. S. Lucas, ${ }^{5}$ M. B. Stone, ${ }^{3}$ and D. L. Abernathy ${ }^{3}$ \\ ${ }^{1}$ Instrument and Source Division, Oak Ridge National Laboratory, Oak Ridge, Tennessee 37831, USA \\ ${ }^{2}$ Materials Science and Technology Division, Oak Ridge National Laboratory, Oak Ridge, Tennessee 37831, USA \\ ${ }^{3}$ Neutron Scattering Division, Oak Ridge National Laboratory, Oak Ridge, Tennessee 37831, USA \\ ${ }^{4}$ California Institute of Technology, Pasadena, California 91125, USA \\ ${ }^{5}$ Air Force Research Laboratory, Wright-Patterson Air Force Base, Ohio 45433, USA
}

(Received 25 April 2017; accepted 29 September 2017; published online 31 October 2017)

\begin{abstract}
We present the design and operating characteristics of a vacuum furnace used for inelastic neutron scattering experiments on a time-of-flight chopper spectrometer. The device is an actively water cooled radiant heating furnace capable of performing experiments up to $1873 \mathrm{~K}$. Inelastic neutron scattering studies performed with this furnace include studies of phonon dynamics and metallic liquids. We describe the design, control, characterization, and limitations of the equipment. Further, we provide comparisons of the neutron performance of our device with commercially available options. Finally we consider upgrade paths to improve performance and reliability. Published by AIP Publishing. https://doi.org/10.1063/1.5007089
\end{abstract}

\section{INTRODUCTION}

Active research areas in condensed matter physics and materials science at elevated temperatures include studies of phonon anharmonicity in functional materials, ${ }^{1-8}$ localization dynamics, ${ }^{9-11}$ phonon entropy, ${ }^{12,13}$ and local dynamics of metallic liquids. ${ }^{14}$ The neutron is a weakly interacting probe of matter, allowing researchers to perform neutron scattering measurements on condensed matter systems with samples placed within complicated environments such as cryostats, furnaces, magnets, and pressure cells. ${ }^{15-17}$ Neutron scattering signals are inherently weak, so optimization of sample volume is critically important to experiment success; nevertheless, excess material from the sample environment in the neutron beam increases background levels, which can make signal interpretation difficult. Thus, minimization of material in the beam path, the addition of bulk internal shielding in the form of neutron absorbers, and material choices all play important roles in the design of sample environment equipment. High temperature environments pose particular design challenges, especially when combined with the potential operation of the sample environment in the vacuum environment of a neutron scattering instrument. Additionally, high temperature environments present significant material interaction concerns, including formation of eutectics and alloying of materials, as well as issues with material creep and yield.

Significant advances have been made in high temperature sample environments for diffraction and engineering materials studies, ${ }^{18-23}$ but the reduced scattering signal available for inelastic measurements critical for dynamic studies presents additional design challenges. This manuscript describes the design and characterization of a furnace used to conduct

a)Author to whom correspondence should be addressed: niedzielajl@ornl.gov inelastic neutron scattering experiments at high temperature with the wide Angular Range Chopper Spectrometer (ARCS) at the Spallation Neutron Source (SNS), Oak Ridge National Laboratory. We discuss the development process of the furnace and strategies for background reduction and operational optimization, and present scattering data comparing the furnace in different operational configurations, and comparing the performance of commercially available options. We also briefly discuss sample mounting strategies, Monte Carlo simulations of multiple scattering effects, and potential upgrades for the device.

\section{THE ARCS INSTRUMENT}

ARCS is a high-flux, direct geometry chopper spectrometer operating at incident energies typically between 20 and $2000 \mathrm{meV}^{24,25}$ The ARCS instrument uses linear position sensitive detectors arranged in a cylindrical configuration about the vertical axis centered at the sample. The range of scattering angles measured is between $2.4^{\circ}$ and $136^{\circ}$, and the beam size is $50 \mathrm{~mm} \times 50 \mathrm{~mm}$. The detector array is housed inside of a large vessel that supports a vacuum level down to the $10^{-7}$ Torr range. A large gate valve allows separation of a portion of this vacuum vessel to enable rapid vacuum cycling of the smaller chamber, facilitating sample changes and use of different sample environments. An oscillating radial collima$\operatorname{tor}^{26,27}$ is also used at the instrument, and the impact of this collimator on reducing background levels is discussed in the present text. While the details presented here are specific to the ARCS instrument, we note that it is possible to install the furnace described here at any SNS instrument using a vacuum environment with a flange mount. Instruments that operate with the sample environment not in vacuum, for example, a sample-table on a triple-axis spectrometer, can also make use of these furnaces with the addition of a vacuum shroud. 
TABLE I. Table of data for elements commonly used in the neutron furnace design. Melting point data are taken from Ref. 41 and are given at atmospheric pressure. $\mathrm{P}_{v}$ indicates the vapor pressure at the given temperature; all values for $P_{v}$ are given in Torr, and data are from Refs. 42 and 43. $\sigma_{a}$ is the neutron absorption cross section for neutrons at $25 \mathrm{meV}$, and $\sigma_{s}$ is the neutron scattering cross section for neutrons at $25 \mathrm{meV}$; both are given in barns for the natural abundance and taken from Ref. 44 .

\begin{tabular}{lcccccc}
\hline \hline Element & $\begin{array}{c}\text { Melting } \\
\text { point }(\mathrm{K})\end{array}$ & $\mathrm{P}_{v} 900 \mathrm{~K}$ & $\mathrm{P}_{v} 1500 \mathrm{~K}$ & $\mathrm{P}_{v} 1800 \mathrm{~K}$ & $\sigma_{a}$ & $\sigma_{s}$ \\
\hline $\mathrm{Al}$ & 933 & $5.4 \times 10^{-10}$ & Liquid & Liquid & 0.231 & 1.503 \\
$\mathrm{Cu}$ & 1357 & $1.3 \times 10^{-10}$ & $1.3 \times 10^{-10}$ & Liquid & 3.78 & 8.03 \\
$\mathrm{Ti}$ & 1941 & $1.2 \times 10^{-17}$ & $7.9 \times 10^{-7}$ & $3 \times 10^{-4}$ & 6.09 & 4.35 \\
$\mathrm{~V}$ & 2183 & $7.1 \times 10^{-20}$ & $6.2 \times 10^{-8}$ & $5.8 \times 10^{-5}$ & 4.9 & 5.09 \\
$\mathrm{Nb}$ & 2750 & $8.4 \times 10^{-36}$ & $4.7 \times 10^{-15}$ & $7.2 \times 10^{-11}$ & 1.15 & 6.255 \\
$\mathrm{Ta}$ & 3290 & $1.7 \times 10^{-36}$ & $8.2 \times 10^{-19}$ & $1.8 \times 10^{-14}$ & 20.6 & 6.01 \\
$\mathrm{~W}$ & 3695 & $7.6 \times 10^{-40}$ & $7.5 \times 10^{-40}$ & $6.0 \times 10^{-15}$ & 18.3 & 4.6 \\
\hline \hline
\end{tabular}

\section{FURNACE CONSTRUCTION}

Furnaces have three primary components: a heating element, an interface for sample mounting, and thermal shielding to prevent radiative heat losses. The heating element can be resistive and heat the sample via conduction through direct thermal contact. Alternatively, a conductive metal sheet with a substantial applied current will radiate heat, and this can be exploited to reach high temperatures. Sample mounting devices are adapted to the experiment being performed, but may be constructed in either a cylindrical or flat-plate geometry. Cylindrical sample geometries are better matched to the shape of the ARCS detector array; flat plate geometries allow for a thinner sample face and construction of sample packets out of foil. Sample mounts must be constructed of material that will not react or sag or move at high temperatures, this point is discussed more in Sec. V. Thermal shielding reflects heat back toward the sample position to prevent radiative losses, helps us to maintain a uniform temperature at the sample position, reduces the power required to reach a desired temperature, and protects the instrument vessel.

Design constraints for sample environments for timeof-flight direct geometry chopper spectrometers (DGSs) are different than that of triple-axis reactor-based instruments. For triple-axis spectrometers, the multiple arms of the instrument allow one to collimate the neutron beam substantially to decrease the background due to sample environments. For DGS time-of-flight instruments, the large and predominately open secondary flight path makes scattered beam collimation difficult. Incident beam collimation is also not typically used for DGS time-of-flight instruments.

An additional important design consideration is the neutron-material interaction. In single element cases, background due to incoherent and coherent scattering is easier to understand. Accordingly, niobium and vanadium are commonly used for their favorable neutron properties and hightemperature capabilities. Vanadium metal has a nearly zero coherent scattering cross section in the vicinity of the elastic line, providing a very smooth background at low energy transfers. Niobium has a stronger coherent scattering contribution but can be used at higher operating temperatures. Table I shows the melting point, vapor pressure, and neutron absorption and scattering cross sections for materials used in furnace construction. Table II shows the same data for materials which are commonly used in neutron (specifically indium, cadmium, and zinc) scattering experiments but should be avoided in furnace applications due to melting or low vapor pressure concerns. Note that this latter list is not exhaustive, and care must also be taken to avoid using alloys containing these materials (i.e., brass).

It is strategic for a neutron scattering program to have a range of devices available for temperature dependent studies. ${ }^{17}$ The compromise between background, temperature operating range, and material construction made possible by multiple devices provides flexibility for neutron user programs. Several commercial sample environment devices are available for neutron scattering applications over a range of temperatures. Low-background bottom-loading closed cycle refrigerators

TABLE II. Table of data for elements commonly used in neutron scattering experiments, but that should be avoided with use in a vacuum furnace. Melting point data are taken from Ref. 41 and are given at atmospheric pressure. $\mathrm{P}_{v}$ indicates vapor pressure at the given temperature; all values for $P_{v}$ are given in Torr, and data are from Refs. 42 and 43. $\sigma_{a}$ is the neutron absorption cross section for neutrons at $25 \mathrm{meV}$, and $\sigma_{s}$ is the neutron scattering cross section for neutrons at $25 \mathrm{meV}$; both are given in barns for the natural abundance and taken from Ref. 44 . This list is not exhaustive, and alloys or devices containing these materials (i.e. brass, materials electroplated with cadmium) should also be avoided.

\begin{tabular}{lcccccc}
\hline \hline Element & $\begin{array}{c}\text { Melting } \\
\text { point (K) }\end{array}$ & $\mathrm{P}_{v} 300 \mathrm{~K}$ & $\mathrm{P}_{v} 500 \mathrm{~K}$ & $\mathrm{P}_{v} 700 \mathrm{~K}$ & $\sigma_{a}$ & $\sigma_{s}$ \\
\hline $\mathrm{In}$ & 430 & $5.4 \times 10^{-10}$ & Liquid & Liquid & 193.8 & 2.62 \\
$\mathrm{Cd}$ & 595 & $3.1 \times 10^{-11}$ & $1.6 \times 10^{-3}$ & Liquid & 2520 & 6.5 \\
$\mathrm{Zn}$ & 693 & $2.5 \times 10^{-14}$ & $2.7 \times 10^{-5}$ & Liquid & 1.11 & 4.5 \\
\hline \hline
\end{tabular}


(CCRs) as configured have a maximum operating temperature of $325 \mathrm{~K}$; hot-stage devices that employ a sapphire thermal switch are available to support operation up to either $450 \mathrm{~K}$ or $800 \mathrm{~K}$. A top-loading device with a special stick equipped with a heater block for a maximum operating temperature of $750 \mathrm{~K}$ is also available commercially. The top-loader limitation is driven by the construction of the device, which employs interior indium seals, and temperatures in excess of $325 \mathrm{~K}$ may weaken these seals. Higher temperatures are accessible in the top loading device with evacuation of all exchange gas from the device, though this results in thermal gradients across the sample. Standard liquid helium cryostats or so-called "dry" pumped helium systems restrict operation to less than $320 \mathrm{~K}$. The operation of liquid helium cryostats relies on a large volume of cold gases, and heating samples to high temperatures can result in dangerous over pressure situations and the failure of internal components. Aerodynamic ${ }^{28-30}$ and electrostatic $^{31,32}$ levitators may provide operation up to $3200 \mathrm{~K}$, but there are stringent sample requirements, and reduced sample masses can be used for experiments. Having additional high temperature sample environment options with optimized backgrounds, thermal stability, and reliability allows for improved measurements without compromising the goals of the experiment.

\section{RADIATIVE HEATING FURNACE}

The furnace we describe uses the radiant heating principle and is known as the MICAS furnace. The MICAS furnace operates between ambient temperatures and $1873 \mathrm{~K}$ in vacuum. A schematic of this device is shown in Fig. 1. This furnace has been used in the study of metallic glasses, ${ }^{2}$ silicon, ${ }^{3}$ yttrium-stabilized zirconia, ${ }^{5} \mathrm{Ag}_{2} \mathrm{O},{ }^{7}$ and $\mathrm{Pt} .{ }^{13}$

The active heating elements are interchangeable and are constructed of either two nested niobium or vanadium foil cylinders with low intrinsic resistance. The inner cylinder of the element has an internal diameter of $80 \mathrm{~mm}$ with a foil thickness of $0.05 \mathrm{~mm}$. The cylinders are nested and tack welded to niobium rings at the top and bottom of the cylinder, and the two cylinders are electrically isolated from each other with the use of ceramic standoffs. The inner sample space has a diameter of $70 \mathrm{~mm}$. From the mounting flange to the beam position, it is $749.3 \mathrm{~mm}$, and the device extends an additional $215.9 \mathrm{~mm}$ below the nominal beam position for an overall in-vacuum length of $965.2 \mathrm{~mm}$ (Fig. 2).

Electrical connections for the heating element are supplied from the top of the unit. Power is supplied via cables threaded out of the top of the furnace unit using 3/O AWG electrical cables. The cables are connected to vacuum tight

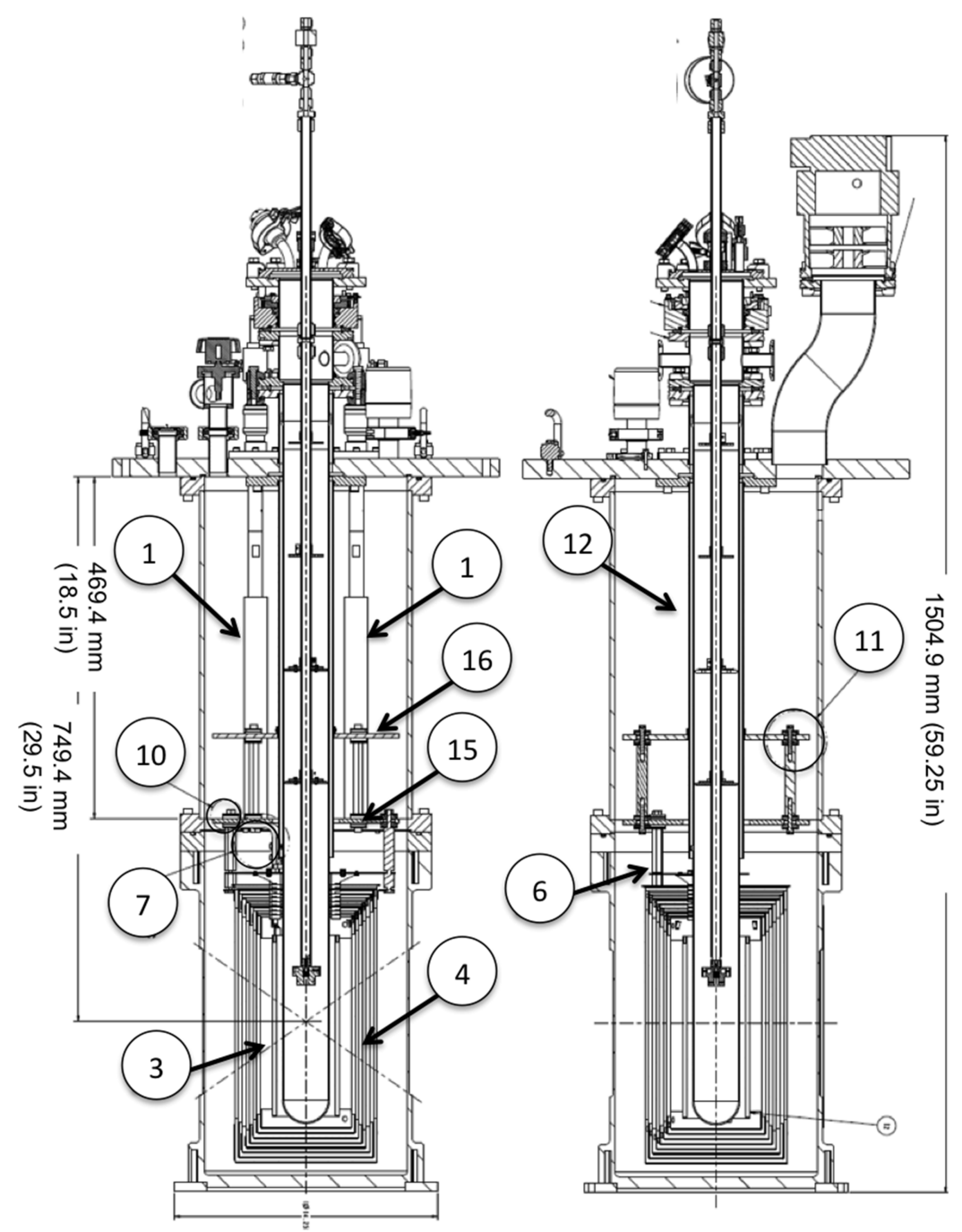

FIG. 1. Engineering drawings of the MICAS overall design, showing the full device length, sample position in the device. All dimensions given in $\mathrm{mm}$ (inches in parentheses). The sample position is marked by the crossed hatched lines at a distance of $490.4 \mathrm{~mm}$ (29.5 in.) from the mounting flange. Copper conductors encased in ceramic sheaths are indicated by (1) and make direct electrical connection to the conducting copper plate (15). The conducting plate (15) is held by a guide plate (16) which is electrically isolated by ceramic standoffs (10 and 11). The copper conducting plate makes the electrical connection to the heating element (3) via copper mounting brackets (7). Concentric nested heat shields (4) are mounted to a bracket (6) and surround the sample position to reflect heat back to the sample. The inner support column (12) is stainless steel. The copper angle bracket (7) is attached to the copper plate (15) by copper screws and washers. All data referenced from Ref. 40. 


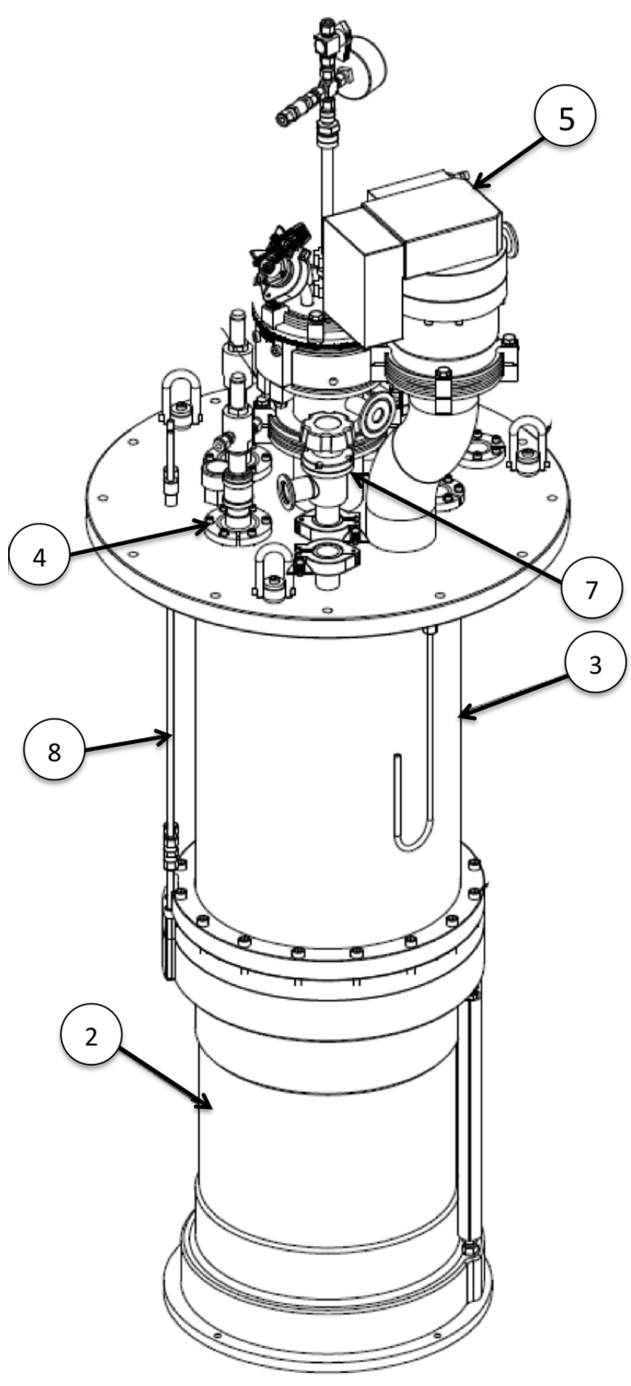

FIG. 2. Isometric view of the exterior of the MICAS furnace. The outer body (3) is stainless steel. The thin window around the sample position (2) is aluminum. The power feedthroughs are indicated at (4), and the turbo pump location is given at (5). Stainless steel water lines (8) thread down the outside of the MICAS before making entry to the interior of the device. The clearance boundary for the whole device is $16 \mathrm{in}$. (406.4 mm), which is the standard diameter boundary of the mounting flange at the SNS. The whole of the MICAS fits within this diameter, with and mates with a stainless steel vacuum flange. A fluoroelastomer O-ring is used to make the high vacuum seal required for operation of the furnace in the vacuum environment of the instrument. A valve for manual venting of the space is shown at (7). All information is taken from Ref. 40.

power feedthroughs on the top of the furnace. The electrical cables are then connected to copper rods with diameter $19 \mathrm{~mm}$ extending down to a $\mathrm{Cu}$ plate where the heating element is mounted. Two angular copper brackets extend downward from the $\mathrm{Cu}$ plate, attached to the $\mathrm{Cu}$ plate with stainless steel screws. Each bracket connects to a niobium leg $1.5 \mathrm{~mm}$ thick, $9.5 \mathrm{~mm}$ wide, and $127 \mathrm{~mm}$ tall. One niobium leg connects to the outer cylinder of the element, and one niobium leg connects to the inner cylinder of the element, and each leg is arc-welded to the rings at the top of the element. Thus the current flow through the element is downward through one cylinder and upward through the other. The heating element is electrically isolated from the furnace body and ground. We note that ceramic pieces are epoxied onto the $\mathrm{Nb}$ legs to prevent the legs from making electrical contact with the heat shields, and also between the two rings at the top of the heating element cylinders.

Surrounding the thermal heating elements are a variable number of nested heat shields which are used to improve the thermal performance. In standard operations, five vanadium shields provide a maximum operating temperature of $1473 \mathrm{~K}$, and eight niobium shields provide $1873 \mathrm{~K}$. Operating at $1873 \mathrm{~K}$ consumes $1.5 \mathrm{~kW}$ with 8 niobium heat shields installed. The power required to maintain the sample temperature increases inversely with the number of thermal shields. ${ }^{33}$ The required type and number of shields installed are determined in advance of the neutron scattering experiment in consultation with the user science program in order to minimize the number of shields and therefore the background. For $8 \mathrm{Nb}$ shields, the shields and heating element represent $1 \mathrm{~mm}$ combined thickness of material for a neutron to penetrate traveling from the source to the sample. Figure 3 shows the difference in background for the case of 4 or 8 niobium shields. Figures 5(b) and 5 (c) show the background from the furnace for the case of 8 niobium and 5 vanadium shields, respectively.

The outgassing of furnace components may affect device performance in situ. Therefore, when not in use, the shield materials are stored in a nitrogen dry box to minimize oxidation and improve outgassing performance. Before being used for an experiment, the furnace is typically assembled into its operational configuration and heated to a mid-range temperature to outgas the components. This process will typically take place two days prior to the installation at the beamline.

The MICAS is a top-loading device, meaning the equipment is installed on the beamline, and samples are mounted onto extendable rods that are then inserted into the top of the furnace. The sample stick for the MICAS was designed to have an adjustable height, allowing flexibility in the design of sample cans and mounts. The sample stick is also equipped with a series of radiation baffles to maintain sample temperature and maintain the sample in the center of the beam. The interior bore of the MICAS device and the fixed height of the beam path relative to the furnace dictate the sample positions, and

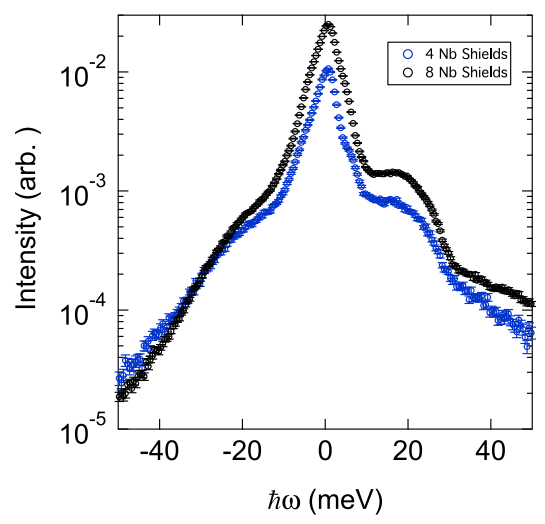

FIG. 3. Comparison of scattered neutron intensity from empty $\mathrm{Nb}$ packet in MICAS with $4 \mathrm{Nb}$ shields and empty $\mathrm{Nb}$ packet in MICAS with $8 \mathrm{Nb}$ shields using an incident energy of $60 \mathrm{meV}$ with the ARCS instrument. Data are presented by integrating over all values of momentum transfer. 
the stick may be made longer or shorter as needed to center the sample material in the beam profile. The sample stick is constructed of a central $\mathrm{Nb}$ rod of diameter $12.7 \mathrm{~mm}$ which is surrounded by a concentric stainless steel outer tube. The niobium rod is free to move in the central tube and is held in place at the top of the device with a stainless steel locking nut. To reduce radiative heat losses through the sample stick, $20 \mathrm{~cm}$ of the sample stick closest to the heating element is wrapped in the tantalum foil that is tack welded to a stainless steel collar. Six thermal radiation baffles constructed of niobium are mounted on the stick at a level starting with the top of the heat shield assembly. The shields are held in place with tabs cut from the tantalum foil surrounding the sample stick. Above the tantalum foil is a stainless steel assembly which connects the stick to an ISO100 vacuum flange.

As mentioned previously, the ARCS sample and detector chambers are able to be isolated from one other via the use of a large stainless steel gate valve. Closing the gate valve permits sample environment changes to be performed without venting the entire detector chamber. In operation, the MICAS furnace shares a common vacuum space with the vacuum of the sample chamber at ARCS and requires a pressure on the order of $10^{-4}$ Torr or better. Sharing the vacuum space with the instrument helps keep the background low but requires venting of the entire sample chamber vacuum space for sample changes. The MICAS has a dedicated turbo pump. The outer wall of the MICAS furnace is an aluminum cylinder $3.175 \mathrm{~mm}$ thick with an angular cutout to accommodate the ARCS detector range; this cutout is covered with a niobium foil of thickness $0.05 \mathrm{~mm}$ which is held in place with aluminum clamps above and below the cutout. ${ }^{34}$ The outer wall modifications are shown in Fig. 4(b). Due to the shared vacuum space, the niobium foil is not sealed. If needed, aluminum foil may be substituted for the niobium foil.

The very thin window provides for a very low background but presents an operational vulnerability when performing sample changes. Venting of the vacuum chamber must be carefully controlled to avoid crumpling the outer wall of the furnace. The temperature of the furnace should be as low as possible before beginning venting to reduce the risk of damage to the materials in the device, for example, by oxidation of the heating element. Consequently, venting is not started until the temperature of the furnace is below $573 \mathrm{~K}\left(300^{\circ} \mathrm{C}\right)$, where the furnace heat loss due to radiative cooling slows down. At that point, the cool down process may be assisted by using a nitrogen backfill and rough vacuum pumping procedure. This procedure helps lower the temperature of the furnace without oxidizing the heat shields. Final venting to atmosphere using dry air is performed when the furnace temperature is less than $475 \mathrm{~K}\left(180^{\circ} \mathrm{C}\right)$, using a controlled flow of dry air into the sample chamber. With air still flowing into the unit, the sample stick may then be removed. At the end of this procedure, the furnace and sample are usually about $373 \mathrm{~K}$ $\left(100^{\circ} \mathrm{C}\right)$.

The control rack was designed at the SNS and contains a DC power supply, a temperature controller, an over temperature sensor, a turbo pump controller, and vacuum gauges. Integrated latching interlocks monitor the pressure, chilled water supply, and temperature of the unit. An emergency stop is implemented on the control rack to rapidly cut power to the heater elements. The power supply supports operation up to $1873 \mathrm{~K}$, and temperature ramping capabilities are frequently used. The control loop uses the proportional-integralderivative (PID) technology. Temperatures of the sample and
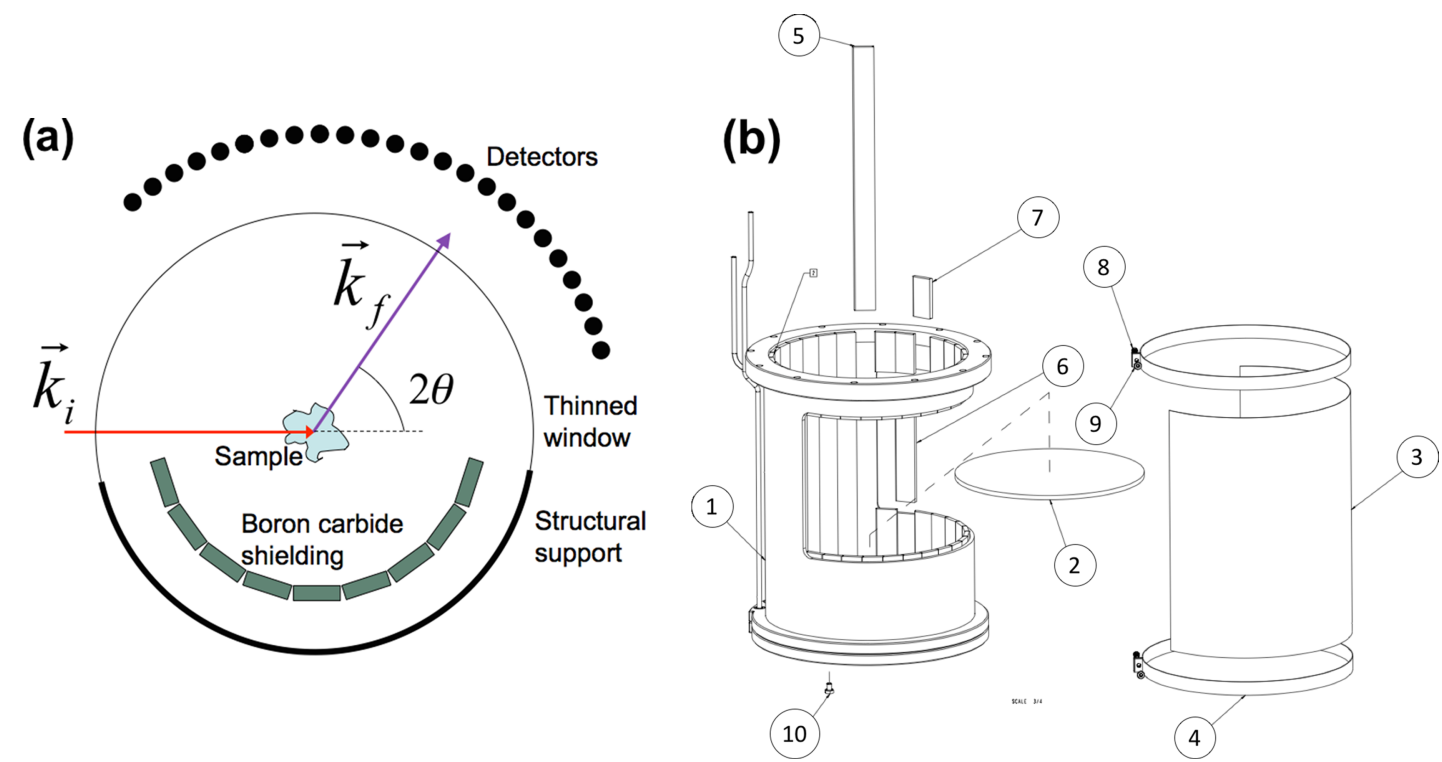

FIG. 4. (a) Schematic showing neutron scattering including the incident beam $\left(k_{i}\right)$, the scattered beam $\left(k_{f}\right)$, and the scattering angle $(2 \theta)$. The detectors are arrayed from $-15^{\circ}$ to $135^{\circ}$ in $2 \theta$. A thin window separates the furnace volume from the detector volume, and this is done to decrease the material the scattered neutrons must transit before reaching the detector position. Boron carbide shielding tiles that efficiently absorb thermal neutrons are installed in the furnace in order to prevent neutrons from scattering off the back wall of the furnace and increasing the background of the measurement. (b) Engineering sketch showing the cutout, thin window, and water lines as actually installed. The outer vacuum wall (OVC) is indicated by (1). The thin window (3) and clamps that constrain the thin window to the furnace body (4) are constructed of aluminum. Boron carbide tiles $(2,5-7)$ are cut to fit the interior wall of the OVC and are epoxied to the inside of the more substantial structural support. (8-10) are locking nuts for the Al clamp rings and base support. 
heat shield are monitored with commercially available type-K or type- $\mathrm{C}$ thermocouples. Type-K thermocouples are easier to install and are used for temperatures less than $1573 \mathrm{~K}$; type-C thermocouples are used for applications requiring a temperature in excess of $1573 \mathrm{~K}$. Power limiting is present on the power supply controller; this limit is typically set to $70 \%$ of the output but can be increased in extreme cases. The power limiting does not trip the power supply off but fixes the supply at the maximal output level. Power is fed into the device via a modified commercial part which mounts the conduction rod which connects to the conducting plate. We also add on a plug adaptor to the top of the feed through. This adaptor is designed to be a cooling jacket as well to remove any excess heat from the power feed through.

Remote control of the furnace is integrated into the SNS instrument data acquisition and control system. A custom built application controls the setting of the device. This application is able to dynamically switch the currently operating PID parameters based on the operating temperature to allow more precise control across different temperature ranges. Programming of the PID tables may be done offline or in situ to increase stability for a given experimental sample. An integrated alarm system alerts staff to alarm conditions, such as a trip of over temperature conditions, to allow rapid response to fault conditions.

When performing sample changes with the MICAS furnace, electrical resistance between the heating elements should be checked to ensure that there is not a short to ground or an open circuit. User procedures developed for the operation of the furnace check for these conditions and also caution against allowing the sample or shields to touch the heating element to avoid burnout of the element or melting of a part of the setup.

To reduce backscattering from the components of the furnace where there are no detectors, a set of boron carbide tiles has been machined from an unfired pre-cursor to reaction bonded boron carbide (RBBC) and mounted over the "dark angle" of the equipment, as shown in Fig. 4. This includes complete blockages in the direction where there is no detector coverage, and partial blockage above the thin window cutout. Further reductions in background were realized when an oscillating radial collimator was installed at the ARCS instrument. The oscillating collimator is specifically designed to reduce the scattering from the material located outside of a gauge volume of the instrument defined by the size of the incident neutron beam. Full details on the design of the oscillating radial collimator are given in Refs. 26 and 27. Figure 5(d) shows the reduction in background of the MICAS furnace afforded by the radial collimator for the furnace with 8 installed niobium heat shields.

Active water cooling of the device is performed with a high throughput standalone chiller and is designed to maintain the temperature of the outer wall as close to room temperature as possible. The temperature at the outer wall as a function of furnace set point up to $1873 \mathrm{~K}$ was tested, and the outer wall temperature at farthest position from the water lines is systematically higher, with a maximum difference of $30 \mathrm{~K}$ at the highest tested temperature. The water lines are stainless steel and are positioned in the "dark angle" of the spectrometer as illustrated in Fig. 4(a), so that no water lines are in the path of the incident or scattered neutron beam. The water lines are also shielded from the scattered neutron beam by the $\mathrm{B}_{4} \mathrm{C}$ tiles in the outer wall. The chiller for the water lines is positioned outside of the sample room, allowing heat to be exhausted to the larger volume of the building of the entire facility, rather than the smaller building which houses the spectrometer. This helps us to avoid overheating of equipment.

Thermal performance of the furnace in the niobium configuration was tested with a vanadium powder sample housed in a vanadium can at the sample position. Temperature control was tested from 540 to $1700 \mathrm{~K}$. A thermocouple was inserted into the sample inside of the can to monitor the sample temperature and to determine the offset from the control temperature. The measured temperature at the sample position follows a linear dependence on the control temperature given by $T_{\text {samp }}\left[{ }^{\circ} \mathrm{K}\right]$ $=1.04 T_{\mathrm{ctl}}+7.53$. Measurement of the equilibration time in this configuration showed that there was no additional equilibration time required for the sample temperature to stabilize when using a ramp rate of $2 \mathrm{~K} / \mathrm{min}$.

One of the advantages of the large angular range scattering geometry of modern time-of-flight spectrometers is the ability to simultaneously collect data over wide regions of reciprocal space. The ability to rotate the sample either in discrete steps or continuously represents an advance in data collection, enabling collection of full dispersion data for condensed matter systems. The MICAS furnace is able to meet this need for single crystal experiments with a rotation stage collar mounted on the sample stick inserted into the furnace. The rotation stage rotates the sample stick freely about the vertical sample axis, and position information from an encoder on the rotation stage
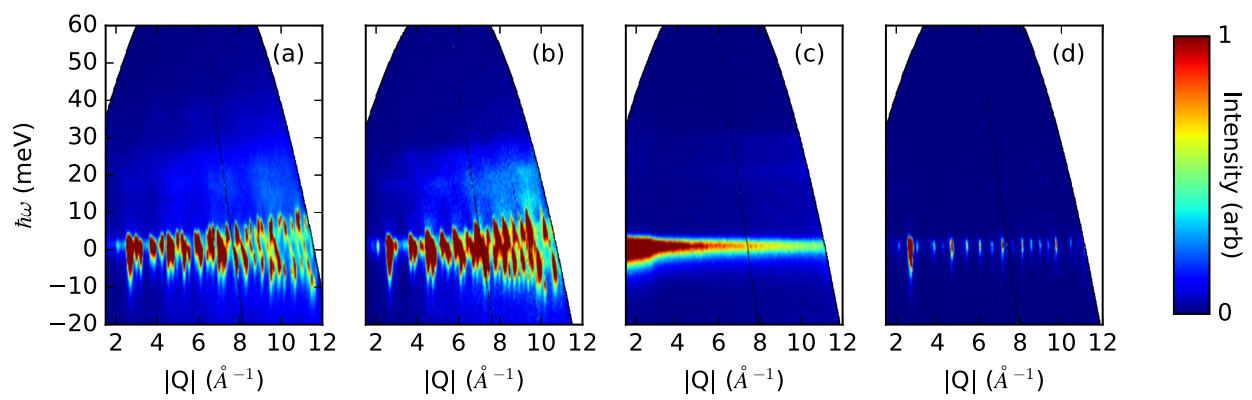

FIG. 5. Comparison of scattered neutron intensity from a niobium packet from the (a) empty commercial furnace, (b) empty MICAS furnace with 8 niobium heat shields, (c) empty MICAS furnace with 5 vanadium heat shields, and (d) empty MICAS furnace with 8 niobium heat shields and the collimator installed. White regions have no data. Measurements are made with $E_{i}=80 \mathrm{meV}$ (a), $60 \mathrm{meV}$ (b), $70 \mathrm{meV}$ [(c) and (d)]. Colors are shown on a linear scale. 
collar is transmitted to the data files. The MICAS furnace can be used with a variety of sample mounts and configurations. The sample stick has an m8-1.25 female sample mounting interface. In MICAS, the most common used sample containers are vanadium sample cans, niobium packets, or niobium sample mounts. To allow for an exchange gas or containment of off-gassing vapors, a quartz tube insert has been designed and allows operation of the furnace up to $1273 \mathrm{~K}$. The quartz assembly consists of a quartz sample stick and two concentric quartz tubes. Thermocouples are fed down the center tube to read the temperature at the sample position, and the sample size is restricted to a diameter of $33 \mathrm{~mm}$. A schematic of the quartz tube assembly and neutron scattering from the empty quartz tube in the furnace are shown in Fig. 6.

We have conducted a comparison of our custom built furnace with a commercially available furnace. Figure 5 shows the scattered neutron intensity from an empty sample container placed within a commercial furnace, and within the MICAS with a thin cut out window and 5 vanadium or 8 niobium shields installed. The commercial furnace has a maximum temperature of $1373 \mathrm{~K}$ and has a comparable elastic line background level to the MICAS furnace when the thin cutout window and $\mathrm{B}_{4} \mathrm{C}$ tiles are installed; however the MICAS furnace has better performance at inelastic energy transfers due to the overall reduction in the material in the path of the neutron. This commercial furnace was designed for triple axis spectrometers, which have different design considerations. Additionally, the commercial furnace sample space is limited to $50 \mathrm{~mm}$, while the design presented here has a $70 \mathrm{~mm}$ sample space, which enables a larger sample volume to be used in the MICAS.

Several design improvements are considered for the MICAS furnace. One would be to replace the interior $\mathrm{B}_{4} \mathrm{C}$ shielding with a material that is less prone to water absorption; this would cut down the time required for outgassing and bake out before an experiment could begin. Another is to improve the heat shield installation assembly. Currently the heat shields are stacked one inside the other and supported

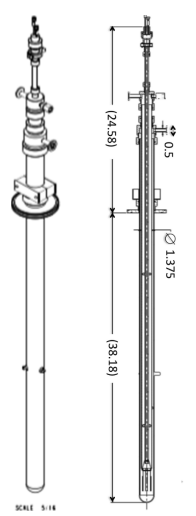

(a)

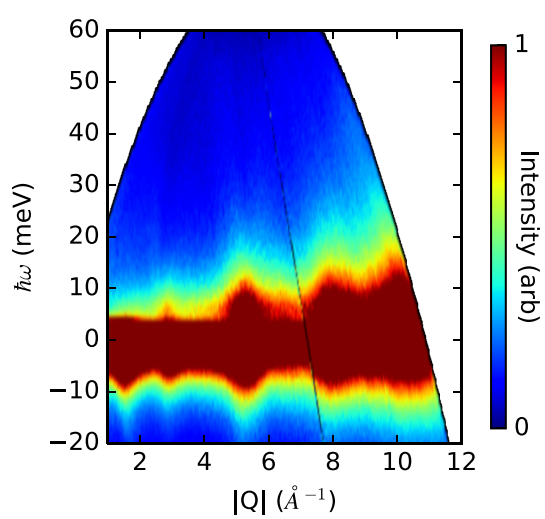

(c)
FIG. 6. (a) Isometric view of the quartz tube insert used in the MICAS furnace. (b) Schematic of the quartz tube insert, all dimensions in inches. (c) Scattered neutron intensity from the empty quartz tube installed in the MICAS with the collimator up and oscillating. The vanadium heating element and 5 shields are installed for this measurement, which is taken with $E_{i}=70 \mathrm{meV}$. Colors are shown on a linear scale. from the top interior ring of the outermost shield using a screw mounting system. Creating a setup with one mounting point that employs a twist-lock configuration rather than screw mounting will make shield installation and removal easier. Improvements to the ceramic standoffs are also planned to remove an operational vulnerability where the ceramic cracks and results in shorting of the element. The ceramic standoffs for the niobium legs for the electrical connection are to be improved in order to remove the need for epoxy.

\section{SAMPLE MOUNTING}

Working with samples at high temperatures presents several unique material challenges, including eutectic formation, thermal yielding, embrittlement due to thermal cycling, and sample vaporization. We briefly address each of these challenges in this section.

In considering materials to use for sample mounting purposes, eutectic formations are a crucial concern. A eutectic refers to a phase mixture where the system melts as a whole at much lower temperature than its constituent parts. An example is a binary alloy of aluminum and germanium, which at a $30 \%$ germanium ratio has a melting point of $693 \mathrm{~K}$, much lower than the melting temperature of either aluminum $(933 \mathrm{~K})$ or germanium $(1211 \mathrm{~K})$. For each measurement performed, potential eutectic formation should be investigated between the sample materials and the container and between the container and the mounting surface of the furnace. Similarly, when the furnace is being designed these potential eutectics of the components should be investigated. The development of a eutectic can result in the melting of the sample can followed by a sudden drop of the sample from the field of view of the neutron beam, along with other deleterious effects. Eutectic formation of binary alloys is fairly easy to foresee from standard phase diagrams ( such as that available from $\mathrm{ASM}^{35}$ ), but ternary and higher alloys require more consideration, and materials must be selected with care. For this purpose, sample cans made from vanadium or niobium are frequently used due to the relative resistance of both of these materials to eutectic formation.

An additional concern in sample mounting is in the yield strength of materials at high temperatures. In particular, stress from thermal cycling can reduce the material strength, resulting in unexpected failure of sample holders, or sudden shifting of sample positions. Also, thermal cycling can increase embrittlement, making sample removal difficult after the neutron experiment.

For furnace applications that require a particular atmosphere inside of the sample can, we have developed a thinwalled aluminum can with a knife edge groove that allows the placement of a thin aluminum foil to act as a sealing medium. The can lid has a knife-edge protrusion that mates to the can body, and a thin aluminum foil may be placed between the two to form the seal. Use of this can in the sealed state is restricted to less that $700 \mathrm{~K}$ due to weakening of the aluminum at high temperature and the increase in the pressure of the exchange gas with temperature that results in deflection of the can walls.

A variety of commercial fasteners are available to fix samples into position. Stainless steel studs will work up to 
moderate temperatures but can gall or have constituents that become miscible at high temperatures or result in eutectic formation at the material boundaries. Molybdenum fasteners may be used but seize after thermal cycling. Sapphire fasteners may be used at high temperatures but are very brittle and are difficult to tighten sufficiently. Brass, copper, cadmium, and indium are all metals typically used in low-temperature neutron scattering applications. Due to their low melting points and high vapor pressures, one must be vigilant to avoid intrusion of these metals into the furnace environments.

Removal of all oils from fingerprints and manufacturing is critical to the experiment performance. We have found that the machine oil residue can cause sample can failure to occur at substantially reduced temperatures. When preparing samples and sample containers, all materials must be cleaned with evaporative solvents that do not leave a residue. Ethanol is commonly used for this purpose. Acetone is not recommended due to residual films left on materials.

Finally, under high temperature conditions in the vacuum environment, samples can vaporize and plate the interior of the furnace. Precursor tests to assess the stability of sample phases are recommended to ensure that the system will be stable over the time scale of the neutron scattering experiment, and containment of suspected off-gassing products is required for materials known to have low sublimation temperatures or vapor pressures. An element of particular concern is zinc, the vapor pressure of which is so low that it will coat the interior of the furnace if any of the components are zinc-plated. Vapor pressure information on commonly used materials in the furnace design is given in Table I and for materials that are common in neutron scattering experiments but not advisable for high temperature experiments is in Table II.

\section{SIMULATIONS FOR NEUTRON SCATTERING}

The furnaces presented herein have undergone an iterative design process to optimize the configuration for high temperature measurement, while attempting to reduce the background contribution from the sample environment in the beam. However, background can provide a considerable contribution to the measured signal, especially for the MICAS furnace. Analysis of the measured data must take into account the contribution of scattering from the sample environment. For powder samples, this is typically done by performing an additional measurement under identical conditions without the sample. ${ }^{45}$ This so-called "empty" measurement is then normalized, and a fraction of this measurement is subtracted from the measurement of the sample. For example, for further reduction of the measured data to a one-phonon density of states, additional multiple scattering and multiphonon corrections are applied. $^{36}$
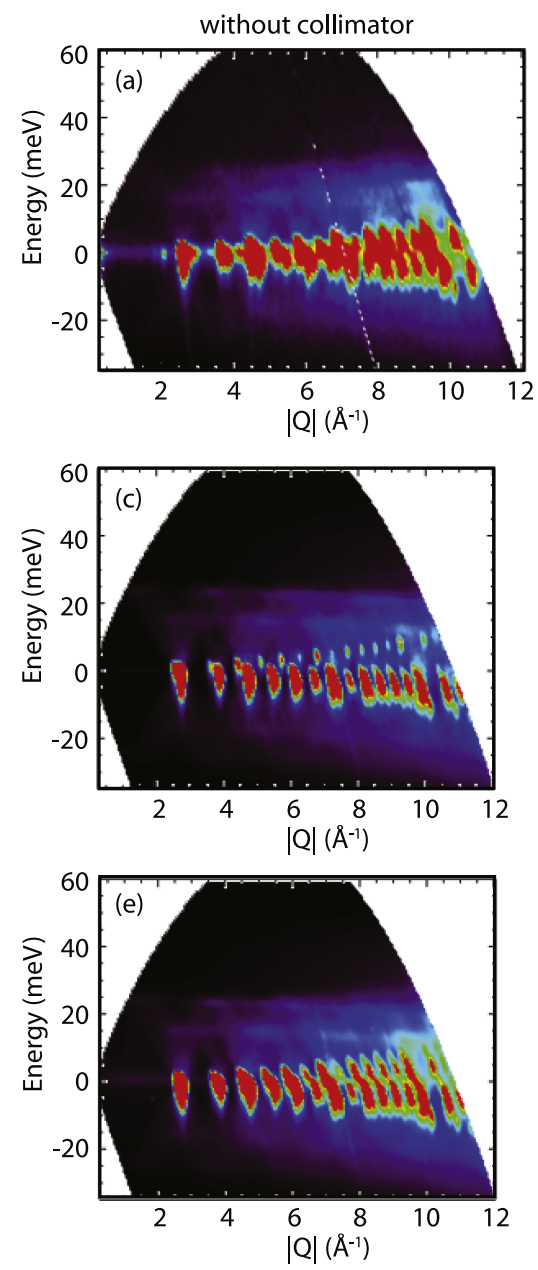
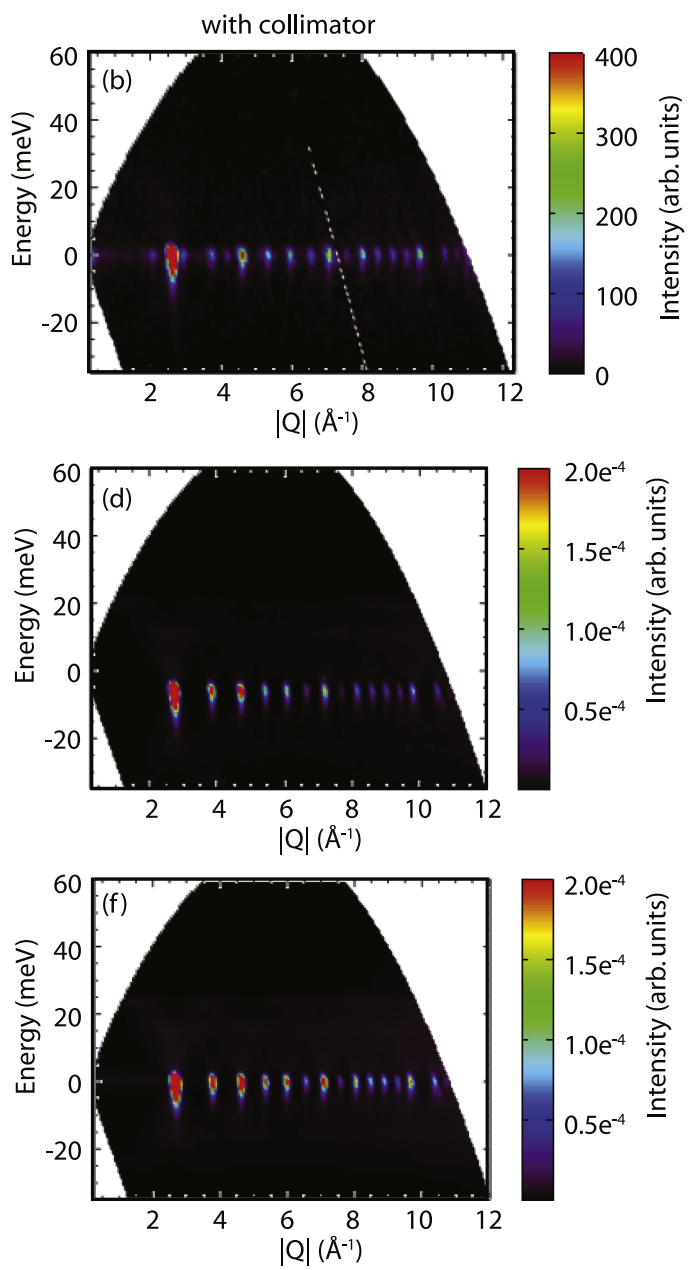

FIG. 7. The experimental results for the MICAS furnace with $8 \mathrm{Nb}$ heat shields at room temperature are given in (a) and (b) for measurement without and with the collimator, respectively. In (c) and (d), the furnace is simulated at room temperature with and without the collimator using scattering kernels for coherent elastic (powder diffraction) and coherent inelastic scattering, incoherent elastic and incoherent single phonon scattering, and multi-phonon scattering using the incoherent approximation. In (e) and (f), all of the kernels in (c) and (d) are used with multiple scattering turned on. Overall the features shown in the experimental data [(a) and (b)] agree well with the simulated data [(e) and (f)]. Comparison of (b) and (d) shows that multiple scattering seems to provide a considerable contribution to the scattered intensity. White areas in the experimental data correspond to physical gaps in the detector coverage. 
To better understand the contribution of scattering from furnace elements to the measured signal, simulations using the Monte Carlo VIrtual Neutron Experiment (MCViNE) package have been employed to model the background contributions for experiments performed in the MICAS furnace. MCViNE is a general purpose Monte Carlo neutron ray tracing package, ${ }^{37}$ used to simulate experimental results from SNS inelastic scattering instruments. ${ }^{38,39}$ An advantage of the MCViNE design architecture is that scattering mechanisms can be selectively enabled to determine how they contribute to the measured scattering intensities. A sample environment module has been constructed from the design specifications of the furnace itself, and this is placed in the beam path for the simulation, concentrically surrounding the sample. Figure 7 compares experimental and simulated plots of $\mathrm{S}(\mathrm{Q}, \mathrm{E})$ for several conditions. The experimental results for the MICAS furnace with $8 \mathrm{Nb}$ heat shields at room temperature are given in (a) and (b) for measurement without and with the collimator, respectively. In (c) and (d), the furnace is simulated at room temperature with a 200 micron-thick $\mathrm{Nb}$ foil $(3.5 \times 4.1 \mathrm{~cm})$, framed with $\mathrm{BN}(1 \times 5 \mathrm{~cm}, 3.4 \mathrm{~mm}$ thick $)$, and placed normal to the beam at the sample position. The simulation with and without the collimator both used the following scattering kernels: coherent elastic (powder diffraction) and coherent inelastic scattering, incoherent elastic and incoherent single phonon scattering, and multi-phonon scattering using the incoherent approximation. In (e) and (f), all of the kernels in (c) and (d) are used with multiple scattering included in the calculation. ${ }^{46}$ Overall the features shown in the experimental data [(a) and (b)] agree well with the simulated data [(e) and (f)]. Comparison of (b) and (d) confirms that multiple scattering from the furnace environment provides a considerable contribution to the scattered intensity. The most obvious difference between (b) and (d) is at the elastic line, which shows that multiple scattering seems to contribute similarly to the signal from incoherent elastic scattering. When the radial collimator is in place [(b), (d), and (f)], the scattered intensity is decreased considerably as compared without the radial collimator [(a), (c), and (e)]. Overall, these simulations validate the effectiveness of the collimator at reducing background signal from the furnace and demonstrate the value of including such calculations to accurately model the sample environment background.

\section{CONCLUSIONS}

In this article, we have described the design, operation, characterization, limitations, and upgrade path for a vacuum furnace used in high temperature inelastic neutron scattering experiments at the Spallation Neutron Source. This equipment has primarily been used on the ARCS time-of-flight chopper spectrometer for inelastic studies but can be extended to use on other instruments at the facility. Future developments will be aimed at improving the reliability of the equipment, and continuing to make improvements in background reduction. Additional developments include improvements of the design of sample holders to reduce background while still maintaining adequate thermal performance. Additional details about the equipment presented here may be found at the SNS sample environment equipment database or by contacting the authors.

\section{ACKNOWLEDGMENTS}

We gratefully acknowledge the contributions of other personnel within the Neutron Sciences Directorate at ORNL including Mariano Ruiz-Rodriguez, Michael Caldwell, Rick Allen, and Ryan Morgan. We also thank B. Fultz of the California Institute of Technology for useful discussion on the furnace design and T. R. Watkins of ORNL for a careful reading of this manuscript. The research at Oak Ridge National Laboratory's Spallation Neutron Source was sponsored by the Scientific User Facilities Division, Office of Basic Energy Sciences, U.S. Department of Energy. This manuscript has been authored by UT-Battelle, LLC under Contract No. DE-AC05-00OR22725 with the U.S. Department of Energy.

This manuscript has been authored by UT-Battelle, LLC under Contract No. DE-AC05-00OR22725 with the U.S. Department of Energy. The United States Government retains and the publisher, by accepting the article for publication, acknowledges that the United States Government retains a non-exclusive, paid-up, irrevocable, world-wide license to publish or reproduce the published form of this manuscript, or allow others to do so, for United States Government purposes. The Department of Energy will provide public access to these results of federally sponsored research in accordance with the DOE Public Access Plan (http://energy.gov/downloads/doepublic-access-plan).

${ }^{1}$ X. Tang, C. W. Li, and B. Fultz, Phys. Rev. B 82, 184301 (2010).

${ }^{2}$ H. L. Smith, C. W. Li, A. Hoff, G. R. Garrett, D. S. Kim, F. C. Yang, M. S. Lucas, T. Swan-Wood, J. Y. Y. Lin, M. B. Stone, D. L. Abernathy, M. D. Demetriou, and B. Fultz, Nat. Phys. 13, 900-905 (2017).

${ }^{3}$ D. S. Kim, H. L. Smith, J. L. Niedziela, C. W. Li, D. L. Abernathy, and B. Fultz, Phys. Rev. B 91, 014307 (2015).

${ }^{4}$ J. W. L. Pang, A. Chernatynskiy, B. C. Larson, W. J. L. Buyers, D. L. Abernathy, K. J. McClellan, and S. R. Phillpot, Phys. Rev. B 89, 115132 (2014).

${ }^{5}$ C. W. Li, H. L. Smith, T. Lan, J. L. Niedziela, J. A. Muñoz, J. B. Keith, L. Mauger, D. L. Abernathy, and B. Fultz, Phys. Rev. B 91, 144302 (2015).

${ }^{6}$ M. S. Lucas, J. A. Muñoz, O. Delaire, N. D. Markovskiy, M. B. Stone, D. L. Abernathy, I. Halevy, L. Mauger, J. B. Keith, M. L. Winterrose, Y. Xiao, M. Lerche, and B. Fultz, Phys. Rev. B 82, 144306 (2010).

${ }^{7}$ T. Lan, C. W. Li, O. Hellman, D. S. Kim, J. A. Muñoz, H. Smith, D. L. Abernathy, and B. Fultz, Phys. Rev. B 92, 054304 (2015).

${ }^{8}$ O. Delaire, A. F. May, M. A. McGuire, W. D. Porter, M. S. Lucas, M. B. Stone, D. L. Abernathy, V. A. Ravi, S. A. Firdosy, and G. J. Snyder, Phys. Rev. B 80, 184302 (2009).

${ }^{9}$ M. E. Manley, D. L. Abernathy, N. I. Agladze, and A. J. Sievers, Sci. Rep. 1, 4 (2011).

${ }^{10}$ M. E. Manley, J. W. Lynn, D. L. Abernathy, E. D. Specht, O. Delaire, A. R. Bishop, R. Sahul, and J. D. Budai, Nat. Commun. 5, 3683 (2014).

${ }^{11}$ M. E. Manley, J. R. Jeffries, H. Lee, N. P. Butch, A. Zabalegui, and D. L. Abernathy, Phys. Rev. B 89, 224106 (2014).

${ }^{12}$ B. Fultz, Prog. Mater. Sci. 55, 247-352 (2010).

${ }^{13}$ Y. Shen, C. W. Li, X. Tang, H. L. Smith, and B. Fultz, Phys. Rev. B 93, 214303 (2016).

${ }^{14}$ T. Iwatshita, D. M. Nicholson, and T. Egami, Phys. Rev. Lett. 110, 205504 (2013).

${ }^{15} \mathrm{G}$. L. Squires, Introduction to the Theory of Thermal Neutron Scattering (Dover, 1978).

${ }^{16}$ G. Shirane, S. M. Shapiro, and J. M. Tranquada, Neutron Scattering With a Triple Axis Spectrometer (Cambridge University Press, Cambridge, 2002).

${ }^{17}$ I. F. Bailey, Z. Kristallogr.-Cryst. Mater. 218, 84 (2003). 
${ }^{18}$ T. Flottmann, W. Petry, R. Serve, and G. Vogl, Nucl. Instrum. Methods Phys. Res., Sect. A 260, 165 (1987).

${ }^{19}$ R. Haynes, A. M. Paradowska, M. A. H. Chowdhury, C. M. Gooday, R. Done, O. Kirichek, and E. C. Olivier, Meas. Sci. Technol. 23, 47002 (2012).

${ }^{20}$ H. M. Kerch, H. E. Burdette, and G. G. Long, J. Appl. Cryst. 28, 604 (1995).

${ }^{21}$ J. Bletry, P. Taverniere, C. Senillou, P. Desre, M. Maret, and P. Chieux, Rev. Phys. Appl. 19, 725 (1984).

${ }^{22}$ S. Katano, H. Motohashi, and M. Iizumi, Rev. Sci. Instrum. 57, 1409 (1986).

${ }^{23}$ R. Winter and T. Bodensteiner, High Pressure Res. 1, 23 (1988).

${ }^{24}$ D. L. Abernathy, M. B. Stone, M. J. Loguillo, M. S. Lucas, O. Delaire, X. Tang, J. Y. Y. Lin, and B. Fultz, Rev. Sci. Instrum. 83, 015114 (2012).

${ }^{25}$ M. B. Stone, J. L. Niedziela, D. L. Abernathy, L. DeBeer-Schmitt, G. Ehlers, O. Garlea, G. E. Granroth, M. Graves-Brook, A. I. Kolesnikov, A. Podlesnyak, and B. Winn, Rev. Sci. Instrum. 85, 045113 (2014).

${ }^{26}$ M. B. Stone, J. L. Niedziela, M. J. Loguillo, M. Overbay, and D. L. Abernathy, Rev. Sci. Instrum. 85, 085101 (2014).

${ }^{27}$ M. B. Stone, J. L. Niedziela, M. Overbay, and D. L. Abernathy, EPJ Web Conf. 83, 03014 (2015).

${ }^{28}$ C. Landron, L. Hennet, J.-P. Coutures, T. Jenkins, C. Aletru, N. Greaves, A. Soper, and G. Derbyshire, Rev. Sci. Instrum. 71, 1745 (2000).

${ }^{29}$ L. Hennet, I. Pozdnyakova, A. Bytchkov, V. Cristiglio, P. Palleau, H. E. Fischer, G. J. Cuello, M. Johnson, P. Melin, D. Zanghi, S. Brassamin, J.-F. Brun, D. L. Price, and M.-L. Saboungi, Rev. Sci. Instrum. 77, 053903 (2006).

${ }^{30}$ G. J. Cuello, V. Cristiglio, L. Hennet, and I. Puente-Orench, J. Phys.: Conf. Ser. 549, 012002 (2014).

${ }^{31}$ H. Aoki, P.-F. Paradis, T. Ishikawa, T. Aoyama, T. Masaki, S. Yoda, Y. Ishii, and T. Itami, Rev. Sci. Instrum. 74, 1147 (2003).

${ }^{32}$ N. A. Mauro, A. J. Vogt, K. S. Derendorf, M. L. Johnson, G. E. Rustan, D. G. Quirinale, A. Kreyssig, K. A. Lokshin, J. C. Neuefeind, K. An, X.-L. Wang, A. I. Goldman, T. Egami, and K. F. Kelton, Rev. Sci. Instrum. 87, 013904 (2016).

${ }^{33} \mathrm{M}$. Wendel and A. Abdou, Initial MICAS Thermal Design, SNS Internal Document (2012).
${ }^{34}$ When in operation, the outer wall on the MICAS is referred to an "outer vacuum jacket" or OVC, though this use is technically incorrect as the outer wall is not vacuum tight. The moniker OVC is in common use at neutron sources, and we elect to use this term when referring to the outer wall on this device, rather than try to create a new term.

${ }^{35} \mathrm{See}$ http://www1.asminternational.org/AsmEnterprise/APD/ for binary phase diagrams.

${ }^{36}$ B. Fultz, T. Kelley, J. Lin, J. D. Lee, O. Delaire, M. Kresch, M. McKerns, and M. Aivazis, Experimental Inelastic Neutron Scattering with a Chopper Spectrometer and Virtual Neutron Scattering with a Computer, http://www.cacr.caltech.edu/projects/danse/doc/Inelastic_Book.pdf (2014).

${ }^{37}$ J. Y. Y. Lin, M. A. Aivazis, and B. Fultz, MCViNE, http://docs.danse.us/ MCViNE (2015).

${ }^{38}$ J. Y. Y. Lin, H. L. Smith, G. E. Granroth, D. L. Abernathy, M. D. Lumsden, B. Winn, A. A. Aczel, M. Aivazis, and B. Fultz, Nucl. Instrum. Methods Phys. Res., Sect. A 810, 86 (2016).

${ }^{39}$ J. Y. Y. Lin, A. A. Aczel, D. L. Abernathy, S. E. Nagler, W. J. L. Buyers, and G. E. Granroth, Phys. Rev. B 89, 144302 (2014).

${ }^{40}$ SNS technical drawing 107030500-M8U-8700-A138, Sample environment MICAS Furnace Furnace Assembly (2010).

${ }^{41}$ CRC Handbook of Chemistry and Physics, edited by David R. Lide (CRC Press, 1996), Chap. 4, pp. 36-98.

${ }^{42}$ CRC Handbook of Chemistry and Physics, edited by David R. Lide (CRC Press, 1996), Chap. 4, pp. 124-125.

${ }^{43}$ C. B. Alcock, V. P. Itkin, and M. K. Horrigan, Can. Metall. Q. 23, 309 (1984).

${ }^{44}$ V.F. Sears, Neutron News 3(3), 26-37 (1992).

${ }^{45}$ Depending on the characterization required, one may additionally use a mock sample with some absorbing characteristics in the sample position for a "dark run" and diamond powder at low incident energies for a characterization of the elastic line, inelastic background, and multiple scattering.

${ }^{46}$ The multiple scattering simulation is truncated at higher orders (at the maximum 8). 